\title{
Treatment of Incidental Prostate Cancer Diagnosed during BPH Surgery with Radical Prostatectomy: Appropriate or over Treatment?
}

\author{
Adnan Simsir ${ }^{1 *}$, Bulent Akdogan ${ }^{2}, \mathrm{Cag} \mathrm{Cal}^{1}$, Sumer Baltaci ${ }^{3}$, Haluk Ozen ${ }^{2}$ \\ ${ }^{1}$ Department of Urology, Ege University School of Medicine, Izmir, Turkey; ${ }^{2}$ Department of Urology, Hacettepe University School \\ of Medicine, Ankara, Turkey; ${ }^{3}$ Department of Urology, Ankara University School of Medicine, Ankara, Turkey. \\ Email: ${ }^{*}$ adnan.simsir@ege.edu.tr, blntakdogan@yahoo.com, cag.cal@ege.edu.tr, sbaltaci@hotmail.com,drhalukozen@gmail.com
}

Received June $2^{\text {nd }}, 2012$; revised July $10^{\text {th }}, 2012$; accepted July $23^{\text {rd }}, 2012$

\begin{abstract}
Objective: Prostate cancer is detected in pathology specimens in 3\% - 16\% of patients undergoing Benign Prostatic Hyperplasia (BPH) surgery. There is no consensus about the optimal treatment plan for these patients. In this study, T1a,b and T1c patients were compared oncologically and in terms of operative morbidity. Materials and Methods: This study included 54 patients (Group 1) undergoing RP based on the detection of incidental prostate cancer and 54 patients (Group 2) undergoing RP based on the diagnosis of carcinoma by biopsy in three different centers. The parameters that may affect the recurrence were investigated and compared between the two groups. Additionally, patient complaints after RP such as stress urinary incontinence, lower urinary tract symptoms and erectile dysfunction were evaluated according to questionnaires and were compared between the groups and within the group in which incidental carcinoma was identified. Data analysis was performed using SPSS version 15. Results: The mean age of the patients was 63.8 years (range $51-78$ years) with a mean follow up of 60.4 months (range $12-82$ months). There were statistical differences in age $(p=0.036)$, pre-RP PSA $(p<0.001)$ and pre-RP prostate volume $(p<0.001)$ between Group 1 and Group 2. Despite the absence of a statistically significant difference in PSM between the two groups $(p=0.09)$, ECE was more common in Group 2 patients $(\mathrm{p}=0.014)$. None of the patients with T1a disease had biochemical recurrence which was observed in 9 patients $(27.3 \%)$ with stage $\mathrm{T} 1 \mathrm{~b}$ disease $(\mathrm{p}=0.007)$. There were significant differences in Gleason scores in BPH specimens and RP specimens, PSM, ECE and T stages between the two subgroups. The assessment of the morbidities that are likely to affect the quality of life by questionnaires revealed that there were no significant differences between Group 1 and Group 2 however significant differences were noted in the erection quality between the subgroups $(p=0.006)$. Conclusion: The course of the disease is determined by the stage of the disease (pTla, pT1b) that indicates tumor burden in these patients. With regard to postoperative complications, the problems affecting the quality of life were not poorer in patients with $\mathrm{T} 1 \mathrm{a}$ and $\mathrm{T} 1 \mathrm{~b}$ disease than that in patients with T1c disease, which is encouraging for radical surgery.
\end{abstract}

Keywords: Radical Prostatectomy; PSA; Biochemical Recurrence; Erectile Dysfunction; Urinary İncontinence; Lower Urinary Tract Symptoms

\section{Introduction}

The interventions performed for the treatment of Benign Prostatic Hyperplasia (BPH) remains to be the most common surgical interventions around the world [1]. Even though the discovery of Prostate Specific Antigen (PSA) is a revolutionary development in the differential diagnosis of BPH and carcinoma, incidental carcinoma is found in pathology specimens in 3\% - $16 \%$ of patients undergoing BPH surgery [2-4]. There is no consensus on what type of treatment should be administered in these patients once

"Corresponding author. diagnosed. While some researchers advocate aggressive treatment, others believe that follow-up would be more appropriate. Radical prostatectomy remains the most effective therapy for the treatment of prostate cancer in patients with a life expectancy of over 10 years [5]. Recently, with increased patient awareness and attempts to increase the quality of life, the aim of cancer surgery has been not only to remove cancer but also to minimize the morbidity rate and to improve quality of life as much as possible. In this study, patients treated with RP based on the diagnosis of incidental prostate cancer (PCA) after $\mathrm{BPH}$ surgery and those who were treated with RP based 
on the diagnosis of PCA made by biopsy with suspicion of clinical PCA were compared oncologically and in terms of morbidity and the necessity and reliability of RP in treatment of incidental PCA were investigated.

\section{Materials and Methods}

In this study, 54 patients who were diagnosed with incidental PCA (T1a,b) by examination of the specimens after BPH surgery and subsequently underwent RP surgery in three different centers between 2004-2010 constituted Group 1. Those patients who underwent ultrasound guided biopsy with suspicion of carcinoma based on PSA level and digital rectal examination (DRE) and subsequently underwent RP after the detection of PCA (T1c) constituted Group 2. While constituting Group 2, the same number of patients with T1c disease $(n=54)$ were included in a retrogade fashion beginning from the operation date of the last T1a,b patient operated. The inclusion criteria were having receiving no neoadjuvant or adjuvant therapies and the study was initiated with a total of 108 patients. Patient data were first analyzed retrospectively. Data derived from medical records of patients included date of operation, time from biopsy and BPH surgery to $\mathrm{RP}$, type of surgery for $\mathrm{BPH}$, patient age, pre-operative PSA, clinical stage, pathologic stage, Gleason scores in biopsy and RP specimens, pre-RP prostate volume, surgical margin positivity (PSM), extracapsular extension (ECE), and history of a nerve sparing surgery. In addition, postoperational PSA levels of patients were examined and time to recurrence in patients with biochemical recurrence, as well as factors affecting recurrence were investigated and compared between the two groups.

Subsequently, all patients were asked to fill out three different questionnaires [ICIQ-SF (International Consultation on Incontinence Questionnaire-short form) for assessment of stress urinary incontinence, IPSS (International Prostate Symptom Score) for assessment of lower urinary tract symptoms and IIEF-5 (International Index of Erectile Function) for assessment of erection quality] that had been previously validated and enabled us to assess the morbidity of RP. After an invitation for follow-up, the questionnaires were filled out by the patients themselves during the follow-up visits, i.e. 60.4 months (12 - 82 months) after radical prostatectomy under supervision of the physicians. The scores obtained were compared between the two groups. All the parameters investigated were also compared within Group 1 (T1a vs T1b) and it was attempted to identify the necessity and reliability of RP in treatment of incidental prostate cancer.

Data analysis was performed using SPSS version 15. T-test, Cox regression analysis, Kaplan-Meier analysis and Mann-Whitney U test were the methods of choice. A $\mathrm{p}$ value of $\mathrm{p} \leq 0.05$ was considered statistically significant.

Despite the results obtained in this study, the reliability of the data obtained based on these questionnaires is limited as these patients did not fill out the questionnaires regularly before RP and thus, pre- and post-RP scores could not be compared. Furthermore, another limitation of this study was the small number of patients enrolled in the study and its non-prospective design (follow up vs radical surgery). However, we believe that, this study, being the first to address a disease exhibiting geographic and racial behavioral differences in a specific region of the world, will be of benefit.

\section{Results}

All patient data were obtained with no difficulty at all. All patients accepted the invitation and filled out the questionnaires. In this study, 108 patients undergoing RP were evaluated. The mean age of the patients was 63.8 years (range 51 - 78 years), with a mean follow-up of 60.4 months (range 12 - 82 months). Table 1 summarizes the characteristics of the patients.

Twenty-seven $(50 \%)$ of 54 patients in Group 1 underwent biopsy at least once with suspicion of PCA before BPH surgery, but were not diagnosed with PCA. Three patients in whom high-grade prostatic intraepithelial neoplasis (HG-PIN) was found on biopsy underwent repeat biopsy and had a biopsy result consistent with BPH and subsequently underwent BPH surgery. It was found that, of 54 patients in Group 1, 21 had a tumor volume of less than 5\% (T1a) in their specimens after BPH surgery

Table 1. Patients characteristics.

\begin{tabular}{lc}
\hline Characteristics & Value (Range or \%) \\
\hline Number of patients & 108 \\
Age (year) & $63.8(51-78)$ \\
Pre RP PSA & $6.30(0.4-25.7)$ \\
Pre RP prostate volume & $38.85(11-105)$ \\
Pre RP Gleason score & $6.27(4-8)$ \\
RP Gleason score & $6.44(5-9)$ \\
Time of RP surgery (week) ${ }^{*}$ & $16.8(5-32)$ \\
Neuron spearing surgery & $34(31.5 \%)$ \\
PSM & $18(17.3 \%)$ \\
ECE & $29(26.9)$ \\
Number of PSA recurrence & $20(18.5)$ \\
Follow-up time (month) & $60.4(12-82)$ \\
ICIQ-SF & $7.61(0-25)$ \\
IPSS & $13.34(1-29)$ \\
IEEF-5 & $9.75(2-24)$ \\
\hline
\end{tabular}

${ }^{*}$ Time between BPH-Radical surgery. 
whereas 33 patients had a tumor volume of above $5 \%$ (T1b) in their specimens. Of these patients, 46 were treated with transurethral resection of the prostate (TUR) for BPH whereas 8 patients were treated with open prostatectomy. Group 1 patients had a mean prostate volume of 62.85 (155 - 23) cc before being operated on for BPH.

\subsection{Oncological Follow-Up}

\subsubsection{Overall Results}

The mean follow up time of the patients was 60.4 (12 $82)$ months. Biochemical recurrence was observed in 20 patients $(18.1 \%)$ at an average of $57.6(18-68)$ months. In the case group, the factors affecting biochemical recurrence were as follows: preoperative PSA level $(p<0.001)$, surgery Gleason score on biopsy-BPH surgery and RP Gleason scores $(p=0.042, p<0.001)$, ECE $(\mathrm{p}=0.036)$, PSM $(\mathrm{p}=0.046)$ and $\mathrm{pT}$ stage $(\mathrm{p}<$ $0.001)$.

\subsubsection{Comparison between Group 1 and Group 2 \\ (Table 2)}

There was no significant difference in follow-up time between the both groups $(p=0.564)$. However, there were significant differences in age $(\mathrm{p}=0.036)$, pre-RP PSA $(\mathrm{p}<0.001)$ and pre-RP prostate volume $(\mathrm{p}<0.001)$ between Group 1 and Group 2. The analysis of pathologic stages of the RP specimens revealed that tumors in Group 2 were in more advanced stages $(p=0.002)$. Despite the absence of a statistically significant difference in PSM between the two groups $(p=0.09)$, ECE was more common in Group 2 patients $(\mathrm{p}=0.014)$.

There was no significant difference in the rate of biochemical recurrence between 9 patients $(16.7 \%)$ in the case group with incidental PCA and 11 patients $(20.4 \%)$ in Group $2(p=0.381)$. Similarly, time to biochemical recurrence did not differ between the groups $(p=0.460)$.

The investigation of the parameters predicting biochemical recurence for the two groups separately revealed that $\mathrm{pT}$ stage (Group $1 \mathrm{p}=0.03$; Group $2 \mathrm{p}<0.001$ ), PSM (Group 1: $11.1 \%, \mathrm{p}=0.04$; Group 2: $22.2 \%, \mathrm{p}<$ 0.001 ) , extracapsular extension (Group 1: 16.7\%, $\mathrm{p}=$ 0.02 ; Group 2: $37.0 \%, p=0.014)$, and Gleason scores (Group: 6.42, $\mathrm{p}<0.001$; Group 2: 6.48, $\mathrm{p}<0.001$ ) were significant predictors for both groups. These data were compared using Kaplan-Meier curves in Figure 1. Additionally, there was no significant relationship between biochemical recurrence and time from BPH surgery to RP ( $p$ $=0.124)$, prostate volumes before BPH surgery and RP $(p=0.178, p=0.383)$ and the type of BPH surgery $(p=$ $0.119)$.

\subsubsection{Comparison between T1a and T1b Patients}

As a result of pathological analysis of the specimens of patients operated on with initial diagnosis of BPH, specimens with a tumor volume of $5 \%$ and below were defined as stage T1a and those with a tumor volume of $5 \%$ and above were defined as stage T1b [6].

There was no statistical difference in the mean follow

Table 2. Comparison of incidental tumors (T1a,b) with T1c tumors (Group 1 vs. Group 2).

\begin{tabular}{|c|c|c|c|}
\hline Parameters & T1a,b & $\mathrm{T} 1 \mathrm{c}$ & $\mathrm{p}$ \\
\hline Number (n) & 54 & 54 & \\
\hline Age $^{*}$ (year) & $64.1(54-73)$ & $62.5(51-78)$ & 0.081 \\
\hline Pre RP PSA ${ }^{*}$ & $3.71(0.4-17.3)$ & $8.69(1.5-25.7)$ & $<0.001$ \\
\hline Prostate weight $^{*}(\mathrm{gr})$ & $25.1(11-52)$ & $53.6(24-105)$ & $<0.001$ \\
\hline After RP Gleason* & $6.42(5-8)$ & $6.48(6-9)$ & 0.242 \\
\hline Number of PSM (\%) & $6(11.1)$ & $12(22.2)$ & 0.09 \\
\hline Number of ECE (\%) & $9(16.7)$ & $20(37.0)$ & 0.014 \\
\hline Neuron spearing prc. (\%) & $7(12.9)$ & $24(50.0)$ & $<0.001$ \\
\hline \multicolumn{4}{|l|}{ After RP pT (\%) } \\
\hline $\mathrm{T} 2$ & $51(94.4)$ & $43(79.6)$ & 0.002 \\
\hline $\mathrm{T} 3$ & $3(5.6)$ & $11(20.04)$ & \\
\hline PSA recurrence $(\%)$ & $9(16.7)$ & $11(20.4)$ & 0.381 \\
\hline PSA recurrence time (month) & $65.1(29-68)$ & $55.9(18-56)$ & 0.460 \\
\hline ICIQ-SF $^{*}$ & $7.48(0-21)$ & $7.75(1-25)$ & 0.269 \\
\hline IPSS $^{*}$ & $14.08(6-29)$ & $12.6(4-28)$ & 0.063 \\
\hline IIEF- $5^{*}$ & $9.11(3-24)$ & $10.39(2-23)$ & 0.19 \\
\hline
\end{tabular}

*: Mean values. 
Group 1 cases
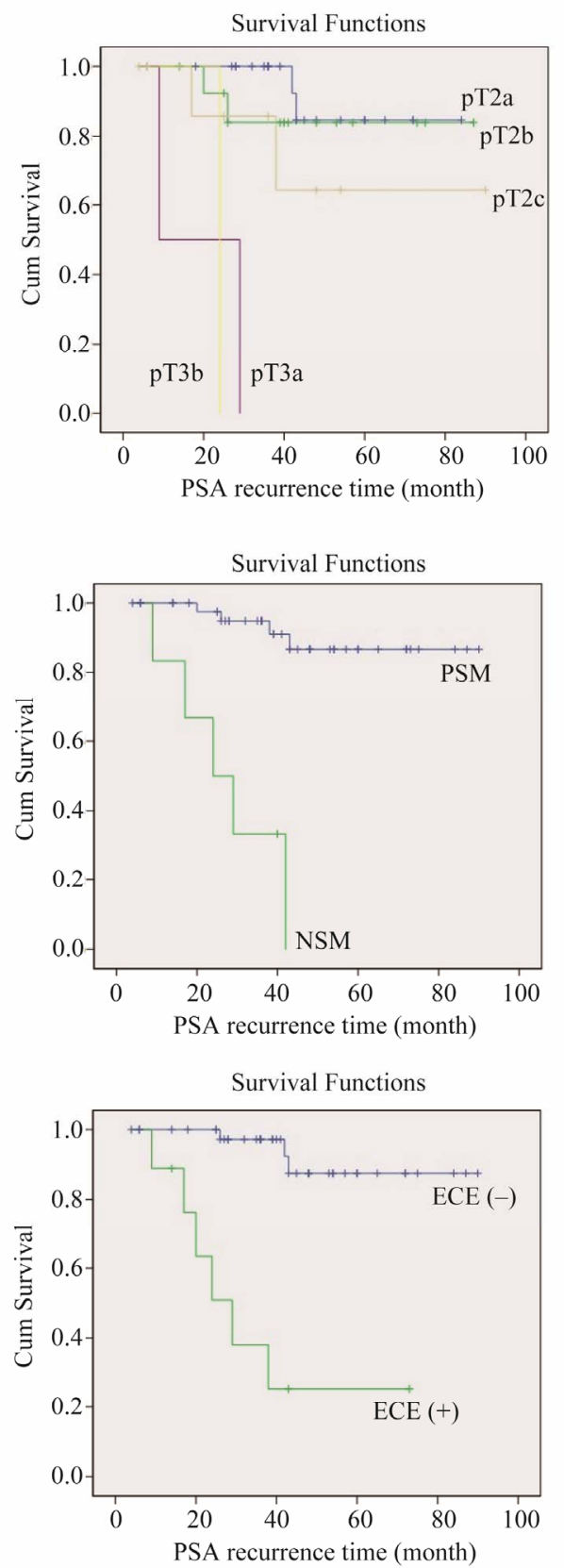

Group 2 cases
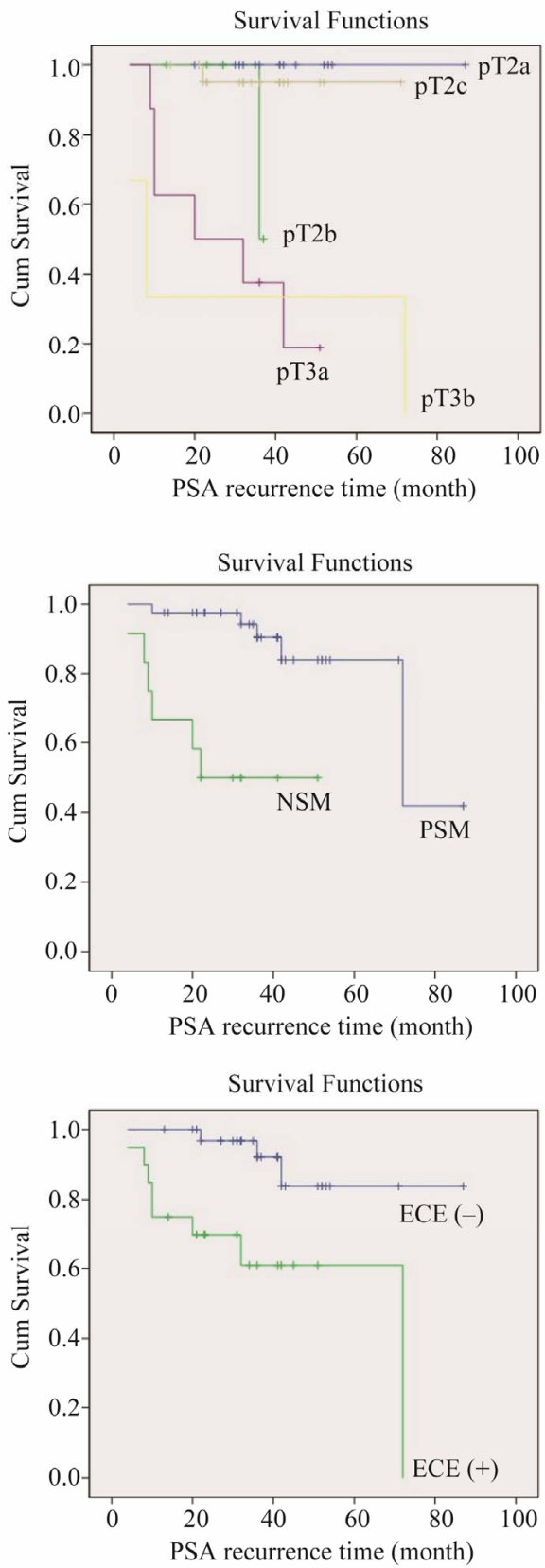

Figure 1. Comparison between the parameters affecting PSA recurrence in Group 1 and Group 2 patients. PSM: Positive surgical margins; NSM: Negative surgical margins; ECE: Extracapsular extansiyon.

up time between pT1a and pT1b patients in Group $1(\mathrm{p}=$ 0.74).

None of T1a patients had biochemical recurrence whereas 9 patients with stage T1b disease $(27.3 \%)$ had recurrence $(p=0.007)$. There were significant differences in Gleason scores in BPH and RP specimens, PSM, ECE and T stages between the two subgroups (Table 3).

There was no significant relationship between the types of BPH surgery (TUR vs. open prostatectomy) and PSM $(p=0.291)$, nerve sparing surgery $(p=0.072)$ and other parameters.

\subsection{Morbidity Follow Up}

\subsubsection{Comparison between Group 1 and Group 2}

There was no statistically significant difference between the groups in stress urinary incontinence after RP assessed 
Table 3. Comparison between T1a and T1b tumors.

\begin{tabular}{|c|c|c|c|}
\hline & $\mathrm{T} 1 \mathrm{a}, \mathrm{b}$ & $\mathrm{T} 1 \mathrm{c}$ & $\mathrm{p}$ \\
\hline Number of patients & 21 & 33 & \\
\hline Age (year) ${ }^{*}$ & $61.6(56-66)$ & $66.8(54-73)$ & 0.061 \\
\hline Pre RP PSA* & $3.2(0.4-11.5)$ & $4.1(0.9-17.1)$ & 0.871 \\
\hline Pre BPH surgery volum ${ }^{*}(\mathrm{cc})$ & $68.2(40-155)$ & $60.1(23-124)$ & 0.024 \\
\hline Pre RP weight ${ }^{*}$ (gr) & $27.6(11-52)$ & $24.6(14-41)$ & $<0.001$ \\
\hline \multicolumn{4}{|l|}{ Type of BPH surgery } \\
\hline TUR & $18(85.7)$ & $28(84.8)$ & 0.421 \\
\hline Open prostatectomy & $3(14.3)$ & $5(15.2)$ & 0.382 \\
\hline RP Gleason score ${ }^{*}$ & $6.38(5-7)$ & $6.48(6-8)$ & 0.041 \\
\hline Number of PSM (\%) & $0(0)$ & $6(18.2)$ & 0.071 \\
\hline Number of ECE (\%) & $0(0)$ & $9(27.3)$ & 0.007 \\
\hline \multicolumn{4}{|l|}{$\mathrm{pT}(\%)$} \\
\hline $\mathrm{T} 2$ & $21(100)$ & $30(90.9)$ & 0.047 \\
\hline $\mathrm{T} 3$ & 0 & $3(19.1)$ & \\
\hline Number of PSA recurrence (\%) & 0 & $9(27.3)$ & 0.007 \\
\hline ICIQ-SF $^{*}$ & $5.95(0-13)$ & $8.45(1-21)$ & 0.260 \\
\hline IPSS $^{*}$ & $11.10(6-19)$ & $16.03(7-29)$ & 0.019 \\
\hline IIEF- $5^{*}$ & $11.19(3-20)$ & $7.79(4-24)$ & 0.006 \\
\hline
\end{tabular}

*: Mean values.

by ICIQ-SF $(p=0.269)$. The comparison of scores on the IPSS assessing lower urinary tract symptoms and the quality of life revealed no difference between Group 1 and Group 2 patients $(p=0.063)$. There was no significant difference when erection quality of the patients was assessed using IIEF-5 $(\mathrm{p}=0.19)$.

It is known that there is an association between neurovasculary bundle injury and morbidities (incontinence, lower urinary tract symptoms, erectile dysfunction) assessed by these three questionnaires. However, the difference between Group 1 and Group 2 patients in terms of the frequency of the use of nerve sparing technique $(12.9 \%$ for Group 1 and $50 \%$ for Group 2, p < 0.001) raises suspicions for the benefits of nerve sparing technique.

\subsubsection{Comparison of T1a and T1b Patients}

The comparison between the two groups revealed no difference in ICIQ-SF $(p=0.260)$ but significant differences in the IPSS $(p=0.019)$ and IIEF-5 $(0.006)$ in favor of T1a (Table 3).

\section{Discussion}

Even though the use of PSA in urological practice has led to important changes in the differential diagnosis of $\mathrm{BPH}$ and PCA, 27\% patients with PSA levels between 0 and $4 \mathrm{ng} / \mathrm{mL}$ have prostate carcinoma [7-10]. Addi- tionally, carcinoma is found in pathology specimens in $3 \%-16 \%$ of patients undergoing BPH surgery $(2-4)$. The impossibility to exclude the presence of PCA based only on the PSA level and the inability to completely exclude PCA to be found incidentally despite all efforts before BPH surgery are two major challenges that had to be overcome. In this study, the primary objectives of this study were to find answers to the following questions: "Should RP be performed in patients in whom incidental PCA was detected after BPH surgery?" and "Does the performance of RP have negative effects on the anticipated morbidity rates in these patients?". The EAU guideline recommends this decision be based on the stage (T1a or T1b) and Gleason score of patients [11]. There was no significant difference in Gleason scores between the cases when grouped as T1a,b and T1c tumors. However, Gleason scores were found to be significantly lower in T1a patients when the cases of stage T1a and T1b were compared in themselves. The absence of biochemical recurrence in patients with stage T1a disease during the follow up period highlights the importance of this finding.

In addition to Gleason score, preoperative PSA levels, surgical margin positivity, clinical and pathologic stage can be used to predict tumor behavior $[12,13]$. The findings obtained by the analysis of data from 108 patients together in the study group support the literature data. 
Even though the comparison between Group 1 and Group 2 patients revealed significant differences in these parameters, the absence of a significant difference in the rates of biochemical recurrence is remarkable. However, this finding can be explained by the fact that all patients with biochemical recurrence in Group 1 were stage T1b and that none of the 21 patients with stage T1a had recurrence. The reason why the subgroups T1a and T1b exhibited such different behaviour lies in the definition of these groups. The tumor volume of the adenoma resected both designates the groups and determines their behaviour. It is well known that prostate cancer frequently arises from the peripheral zone and spreads to the central and transitional zones where the adenoma develops due to increased tumor volume over time. Thus, the more aggressive the tumor is, the higher the volume of the tumor to be extirpated will be as a result of a surgery for adenoma.

With improvements in surgical technique and increasing frequency of RPs, this procedure has become a moderately difficult surgery performed routinely today. From this point of view, can RP surgery be performed in all prostate cancer patients with a life expectancy of more than 10 years? The answer to this difficult question is an issue of concern for both patients and physicians. It can not be ignored that morbidity following RP may lead to social isolation, introversion and major depression in patients [14-16]. RP surgeries are more difficult and longer with a higher amount of blood loss in patients undergoing BPH surgery compared to those in others, which will undoubtedly have an effect on post surgical morbidity $[17,18]$. Surgical margin positivity is the factor that determine the clinical course of the disease by influencing the success of surgery in a difficult RP. The comparison of the study groups revealed that there was no significant difference in PSM between the two groups and that this rate was even lower in Group 1. However, PSM, just like biochemical recurrence, was not observed in T1a patient group, which makes us consider that PSM is associated with tumor burden rather than difficulties during surgery. However, Group 2 patients were at a significantly more advanced pT stage that is one of the most improtant factors influencing PSM, which also supported this interpretation.

The morbidities affecting the quality of life after RP (erectile dysfunction, urinary incontinence, etc.) can be reduced substantially by neurovascular bundle sparing [19]. However, it is crucial that the anatomy of the prostate is closer to normal and not adherent to the surrounding tissues for nerve sparing surgery. Therefore, as seen in this study, nerve sparing surgery could be performed in only $12.9 \%$ of Group 1 patients whereas this rate was very high at $50 \%$ in Group 2 patients. It is evident that a previous history of BPH surgery would substantially complicate neurovascular bundle dissection. Despite the absence of a significant difference between Group 1 and Group 2 based on the results of the questionnaires, it was found that erection quality was poorer in Group 1 patients and that LUTS were more severe in Group 1. The evaluation of Group 1 in itself revealed that erection quality was significantly greater and the severity of LUTS was significantly lower in patients with stage T1a tumors. An important finding is that there was no significant difference in rates of urinary incontinence between T1a and T1b. Even though the risk of erectile dysfunction and LUTS was low in patients undergoing RP with the diagnosis of T1a carcinoma, the incidence of post prostatectomy incontinence is as high as that in patients with stage T1b and T1c disease. Given that incontinence is the most common complication affecting the quality of life after RP, it should be kept in mind that the treatment of patients with stage T1a carcinoma with RP might not be that innocent.

\section{Conclusion}

Despite all improvements in cancer screening before surgery, the incidence rate of incidental prostate adenocarcinoma after BPH surgery remains to be high. The course of the disease is determined by the stage of the disease (pT1a, pT1b) that indicates tumor burden in these patients. Based on data analysis, the approach to patients with T1b PCA should be the same as in patients diagnosed with stage T1c disease. Regarding possible postoperative complications, the problems affecting the quality of life were not worse in patients with stage T1a and T1b disease than those in patients with T1c disease, which is encouraging for radical surgery. Even though T1a tumors exhibit a benign behavior, patient's views should be taken into account in treatment planning and a common decision should be reached.

\section{REFERENCES}

[1] J. Rassweiler, D. Teber, R. Kuntz and R. Hofmann, "Complications of Transurethral Resection of the Prostate (TURP): Incidence, Management, and Prevention," European Urology, Vol. 50, No. 5, 2006, pp. 969-979. doi:10.1016/j.eururo.2005.12.042

[2] H. Lepor, R. S. Owens, V. Rogenes and E. Kuhn, "Detection of Prostate Cancer in Males with Prostatism," The Prostate, Vol. 25, No. 3, 1994, pp. 132-140. doi:10.1002/pros.2990250304

[3] R. M. Merrill and C. L. Wiggins, "Incidental Detection of Population-Based Prostate Cancer Incidence Rates through Transurethral Resection of the Prostate," European Urology, Vol. 7, No. 5, 2002, pp. 213-219. doi:10.1016/S1078-1439(02)00193-X 
[4] D. G. Bostwick, W. H. Cooner, L. Denis, G. W. Jones, P. T. Scardino and G. P. Murphy, "The Association of Benign Prostatic Hyperplasia and Cancer of the Prostate," Cancer, Vol. 70, No. S1, 1992, pp. 291-301. doi:10.1002/1097-0142(19920701)70:1+<291::AID-CNC R2820701317>3.0.CO;2-4

[5] F. J. Bianco Jr., P. T. Scardino and J. A. Eastham, "Radical Prostatectomy: Long-Term Cancer Control and Recovery of Sexual and Urinary Function ('Trifecta')," Urology, Vol. 66, No. 5, 2005, pp. 83-94. doi:10.1016/j.urology.2005.06.116

[6] U. Capitanio, V. Scattoni, M. Freschi, et al., "Radical Prostatectomy for Incidental (Stage T1a-T1b) Prostate Cancer: Analysis of Predictors for Residual Disease and Biochemical Recurrence," European Urology, Vol. 4, No. 1, 2008, pp. 118-125. doi:10.1016/i.eururo.2008.02.018

[7] W. J. Catalona, J. P. Richie, F. R. Ahmann, et al., "Comparison of Digital Rectal Examination and Serum Prostate Specific Antigen in the Early Detection of Prostate Cancer: Results of a Multicenter Clinical Trial of 6630 Men," The Journal of Urology, Vol. 151, No. 5, 1994, pp. 1283-1290.

[8] I. M. Thompson, D. K. Pauler, P. J. Goodman, et al., "Prevalence of Prostate Cancer among Men with a Prostate-Specific Antigen Level < or $=4.0$ ng per Milliliter," The New England Journal of Medicine, Vol. 27, No. 350, 2004, pp. 2239-2246. doi:10.1056/NEJMoa031918

[9] T. A. Stamey, F. S. Freiha, J. McNeal, et al., "Localized Prostate Cancer. Relationship of Tumor Volume to Clinical Significance for Treatment of Prostate Cancer," Cancer, Vol. 71, No. S3, 1993, pp. 933-938. doi:10.1002/1097-0142(19930201)71:3+<933::AID-CNC R2820711408>3.0.CO;2-L

[10] W. J. Catalona, A. W. Partin, K. M. Slawin, et al., "Use of the Percentage of Free Prostate-Specific Antigen to enhance Differentiation of Prostate Cancer from Benign Prostatic Disease: A Prospective Multicenter Clinical Trial," The Journal of the American Medical Association, Vol. 279, No. 19, 1998, pp. 1542-1547. doi:10.1001/jama.279.19.1542

[11] G. Aus, C. C. Abbou, M. Bolla, et al., "EAU Guidelines on Prostate Cancer," European Urology, Vol. 48, No. 4, 2005, pp. 546-551. doi:10.1016/j.eururo.2005.06.001

[12] M. W. Kattan, T. M. Wheeler and P. T. Scardino, "Postoperative Nomogram for Disease Recurrence after Radical Prostatectomy for Prostate Cancer," Journal of Clinical Oncology, Vol. 17, No. 5, 1999, pp. 1499-1507.

[13] G. W. Hull, F. Rabbani, F. Abbas, T. M. Wheeler, M. W Kattan and P. T. Scardino, "Cancer Control with Radical Prostatectomy alone 1000 Consecutive Patients," The Journal of Urology, Vol. 167, No. 2, 2002, pp. 528-534. doi:10.1016/S0022-5347(01)69079-7

[14] R. M. Benoit, M. J. Naslund and J. Cohen, "Complications after Radical Retropubic Prostatectomy in the Medicare Population," Urology, Vol. 56, No. 1, 2000, pp. 116 120. doi:10.1016/S0090-4295(00)00539-2

[15] H. W. Herr, "Quality of Life of İncontinent Men after Radical Prostatectomy," The Journal of Urology, Vol. 151, No. 3, 1994, pp. 652-654.

[16] H. C Klinger and M. Marberger, "Incontinence after Radical Prostatectomy: Surgical Treatment Options," Current Opinion in Urology, Vol. 16, No. 2, 2006, pp. 60-64.

[17] I. M. Thompson and E. J. Zeidman, "Extended Follow up of Stage A1 Carcinoma of Prostate," Urology, Vol. 33, No. 6, 1989, pp. 455-458. doi:10.1016/0090-4295(89)90128-3

[18] K. Bandhauer and E. Senn, "Radical Retropubic Prostatectomy after Transurethral Prostatic Resection," European Urology, Vol. 15, No. 6, 1988, pp. 180-181.

[19] M. V. Catarin, G. M. Manzano, J. A. Nóbrega, F. G. Almeida, M. Srougi and H. Bruschini, "The Role of Membranous Urethral Afferent Autonomic İnnervation in the Continence Mechanism after Nerve Sparing Radical Prostatectomy: A Clinical and Prospective Study," The Journal of Urology, Vol. 180, No. 6, 2008, pp. 2527-2531. doi:10.1016/j.juro.2008.08.020 